\title{
Influencing Factors of Coronary Artery Calcification in Young and Middle-Aged Maintenance Hemodialysis Patients
}

\author{
Shi Zhen, Zheng shu-bei, Jin Ling-wei, Zhou Zhi-hong and Huang Wen* \\ Department of Nephrology, Second Affiliated Hospital \& Yuying Children's Hospital of Wenzhou Medical University, China
}

*Corresponding author: Huang wen, Department of Nephrology, the second affiliated hospital of Wenzhou medical university, Wenzhou 325000, China.

To Cite This Article: Shi Zhen, Zheng shu-bei, Jin Ling-wei, Zhou Zhi-hong, Huang Wen, Influencing Factors of Coronary Artery Calcification in Young and Middle-Aged Maintenance Hemodialysis Patients. Am J Biomed Sci \& Res. 2021 - 12(2). AJBSR.MS.ID.001731.

DOI: 10.34297/AJBSR.2021.12.001731.

Received: February 25, 2021; Published: 制 March 09, 2021

\begin{abstract}
Introduction
Background: Cardiovascular disease has become the main cause of death in patients with chronic kidney disease. Here, we investigated the relationship of coronary artery calcification and the klotho level in young and middle-aged patients of maintenance hemodialysis (MHD) and probed the risk factors of coronary artery calcification. Methods: the clinical data of 64 enrolled patients were collated. Quantification of coronary artery calcification was determined by MSCT for coronary artery calcification score (CACs).
\end{abstract}

Findings: Sixty-four patients (48.44\%) had coronary artery calcification, and fifteen patients (23.44\%) had severe coronary artery calcification Compared with the non-calcification group, IVST, LVPWT and LVMI were higher in calcification group $(\mathrm{p}<0.05)$. Correlation analysis showed that the levels of CACs were related to LVPWT, LVMI. Age, diabetes percentage, male to female ratio, BMI, CRP level were higher in calcification group ( $\mathrm{p}<0.05$ ). Compared with the other two groups, serum albumin, serum Klotho levels were lower, whereas triglyceride, phosphorus, calcium phosphorus product were higher in severe calcified groups $(\mathrm{p}<0.05)$.Correlation analysis showed that the levels of CACs were positively related to age, BMI, serum phosphorus, calcium- phosphorus product, CRP and triglyceride; and negative related to serum albumin, klotho protein. Multiple regression analysis demonstrated that the age, triglyceride were hazardous factors to the CAC, whereas serum albumin, klotho protein were protective factors.

Conclusion: The prevalence of coronary artery calcification is high in young and middle-aged patients of maintenance hemodialysis. Klotho may be a new treatment target for the coronary artery calcification.

Keywords: Young and middle-aged patients, maintenance hemodialysis, Coronary artery calcification score, Klotho protein

\section{Background}

With the improvement of medical level and social development, cardiovascular disease has become the main cause of death in patients with chronic kidney disease. However, the incidence of cardiovascular events was higher in CKD patients on dialysis, and the risk of cardiovascular events was 10 to 20 times higher than that in the general population [1]. Coronary artery calcification (CAC) is one of the independent pathogenic factors of cardiovascular disease in maintenance hemodialysis patients [2]. Therefore, coronary artery calcification can predict the risk of cardiovascular disease in hemodialysis patients. At present, multi-slice spiral CT (MSCT) can effectively evaluate the severity of coronary artery calcification. Klotho gene is a newly discovered anti-aging gene, which can regulate vitamin $\mathrm{D}$, calcium and phosphorus metabolism, protect cardiovascular system, delay aging [3].

In the past, we paid more attention to coronary artery calcification in elderly hemodialysis patients [4]. However, no paper on coronary artery calcification in young hemodialysis patients was published before. In this paper, we will study the coronary artery 
calcification in young and middle-aged maintenance hemodialysis patients and identify the high risks of coronary artery calcification, in order to improve the survival rate and long-term prognosis of young and middle-aged hemodialysis patients.

\section{Objects and Methods}

\section{Objects}

A total of 64 hemodialysis patients who were less than 60 years old and maintained hemodialysis for more than 6 months in the hemodialysis center of our hospital were selected, including 43 males (67.18\%) and 21 females (32.82\%). The average age was $48.63 \pm 8.84$ years, and the average dialysis time was $45.84 \pm 36.32$ months. The primary diseases included chronic glomerulonephritis $(n=30)$, diabetic nephropathy $(n=13)$, hypertensive nephropathy $(n=7)$, polycystic kidney disease $(n=6)$, hepatitis associated nephropathy ( $\mathrm{n}=4)$, aristolochic acid nephropathy $(\mathrm{n}=2)$, lupus nephritis $(\mathrm{n}=1)$ and Alport nephropathy $(\mathrm{n}=1)$.

\section{Exclusion Criteria}

Acute kidney injury, acute infection, malignant tumor, severe malnutrition, severe hepatopulmonary disease and patients who had undergone coronary artery bypass grafting and stent implantation. This study was approved by the Ethics Committee of our hospital, and all patients signed the informed consent form.

\section{Methods}

Coronary Artery Calcification Score Calculation Method: Philips 16-slice spiral CT was used to scan the heart. Four coronary arteries (left trunk, anterior descending branch, circumflex branch and right coronary artery) were scanned respectively. According to the quantitative method of coronary artery calcification of Agaston, the score of coronary artery calcification was obtained. Coronary artery calcification grade: according to Rumberger classification, $0-10$ points as no calcification, $11-400$ points as mild to moderate calcification, $>400$ points as severe calcification.

Biochemical Indexes: $3 \mathrm{ml}$ of fasting blood was collected in the morning, and hemoglobin, C-reactive protein (CRP), albumin, serum creatinine, blood urea, serum uric acid, serum calcium, serum phosphorus, whole segment parathyroid hormone (iPTH), blood triglyceride, total cholesterol, high density lipoprotein, low density lipoprotein, ferritin and other indexes were measured every month for three months, and the averaged values of those three were taken. The concentration of serum secretory Klotho protein was detected by ELISA method. The kit was purchased from Boyao Company, Shanghai.

Determination of Cardiac Structure and Function: all patients were examined by echocardiography within 2 hours after dialysis to avoid the changes of cardiac structure caused by the change of interdialysis volume. The left ventricular end-diastolic diameter ((LVDd)), left ventricular end-systolic diameter ((LVDs)), interventricular septal thickness ((IVST)), left ventricular posterior wall thickness ((LVPWT)), ejection fraction ((E F)) and the ratio of $E$ peak to A peak velocity (Emax A) were measured by machine model SONOS5500NP color Doppler echocardiography. The left ventricular mass index ((LVMI)) was calculated according to Devereux formula.

LVMI= left ventricular weight (LVM)/body surface area (BSA).

LVM =1.04×[(LVDd+IVST+LVPET $) 3-$ LVDd 3]-13.6.

\section{Statistical Methods}

SPSS 17.0 statistical software was used. The measurement data are expressed by mean \pm standard deviation or median (quartile range). The measurement data with normal distribution are analyzed by one-way ANOVA, otherwise are tested by Kruskal-Wallis Test .The counting data were described by the number of cases and percentage, the chi-square test was used for the comparison between groups, and the Bonferroni method was used to test level in-between groups. Pearson was used for correlation analysis. Multiple linear regression was used to analyze the risk factors of CACs. $\mathrm{P}<0.05$ was considered to be statistically significant.

\section{Results}

Coronary artery calcification: the range of CACs in 64 patients was $0-5008.98$, with a median of 5.17 (0365.89). The calcification score was 0 in 29 cases, 0 -10 in 4 cases, $11-400$ in 16 cases and $>400$ in 15 cases. Patients with coronary artery calcification (CACs $>10$ ) accounted for $48.44 \%$, of which mild to moderate calcification accounted for $25 \%$, severe calcification accounted for 23.44\% (Table 1).

Table 1: Comparison of clinical biochemical indexes in three groups of patients.

\begin{tabular}{|c|c|c|c|}
\hline Indicators & Non-Calcification Group & Mild to Moderate Calcification Group & Severe Calcification Group \\
\hline Number of cases & 33 & 16 & 15 \\
\hline age(years) & $44.58 \pm 8.19$ & $55.13 \pm 4.83^{* *}$ & $50.60 \pm 9.05^{* *}$ \\
\hline Male [n (\%)] & $19(57.57 \%)$ & $12(75 \%)$ & $11(73.33 \%)$ \\
\hline Hypertension [n (\%)] & $30(90.91 \%)$ & $15(93.75 \%)$ & $14(93.34 \%)$ \\
\hline Diabetes [n (\%)] & $5(15.15 \%)$ & $8(50.00 \%)^{* *}$ & $8(53.33 \%) *$ \\
\hline Smoking [n (\%)] & $11(33.33 \%)$ & $7(43.75 \%)$ & $6(40 \%)$ \\
\hline
\end{tabular}




\begin{tabular}{|c|c|c|c|}
\hline During of hemodialysis(months) & $48.70 \pm 34.54$ & $32.88 \pm 17.26$ & $53.46 \pm 51.11$ \\
\hline Systolic pressure (mmHg) & $145.78 \pm 30.15$ & $150.24 \pm 28.49$ & $152.35 \pm 27.64$ \\
\hline Diastolic pressure (mmHg) & $85.15 \pm 10.32$ & $82.34 \pm 16.53$ & $87.65 \pm 18.81$ \\
\hline BMI (cm/kg2) & $20.01 \pm 3.34$ & $22.16 \pm 3.69 *$ & $22.17 \pm 2.91^{*}$ \\
\hline $\mathrm{CRP}(\mathrm{mg} / \mathrm{L})$ & $2.82 \pm 1.84$ & $4.26 \pm 2.60^{*}$ & $4.08 \pm 2.10^{*}$ \\
\hline Fer(ng/ml) & $681.26 \pm 229.57$ & $642.92 \pm 398.20$ & $738.57 \pm 254.54$ \\
\hline $\operatorname{Alb}(\mathrm{g} / \mathrm{L})$ & $33.34 \pm 2.19$ & $32.77 \pm 1.73$ & $31.26 \pm 2.96^{* *}$ \\
\hline $\mathrm{HB}(\mathrm{g} / \mathrm{L})$ & $105.16 \pm 7.10$ & $101.5 \pm 2.54$ & $101.56 \pm 12.61$ \\
\hline Scr(umol/L) & $976.38 \pm 190.89$ & $934.08 \pm 244.05$ & $1002.46 \pm 308.89$ \\
\hline BUN (mmol/L) & $457.65 \pm 57.26$ & $435.94 \pm 70.70$ & $467.16 \pm 77.23$ \\
\hline UA (umol/L) & $457.65 \pm 57.26$ & $435.94 \pm 70.70$ & $467.16 \pm 77.23$ \\
\hline $\mathrm{TG}(\mathrm{mmol} / \mathrm{L})$ & $2.26 \pm 1.93$ & $2.30 \pm 1.01$ & $3.61 \pm 2.31 * \#$ \\
\hline $\mathrm{TC}(\mathrm{mmol} / \mathrm{L})$ & $4.11 \pm 0.77$ & $4.45 \pm 1.18$ & $4.37 \pm 1.16$ \\
\hline LDL (mmol/L) & $1.99 \pm 0.59$ & $2.36 \pm 0.89$ & $1.91 \pm 0.39$ \\
\hline HDL (mmol/L) & $0.96 \pm 0.30$ & $0.91 \pm 0.27$ & $0.80 \pm 0.20$ \\
\hline $\mathrm{Ca}(\mathrm{mmol} / \mathrm{L})$ & $2.19 \pm 0.13$ & $2.22 \pm 0.18$ & $2.19 \pm 0.21$ \\
\hline $\mathrm{P}(\mathrm{mmol} / \mathrm{L})$ & $1.79 \pm 0.36$ & $1.77 \pm 0.46$ & $2.08 \pm 0.41 * \#$ \\
\hline $\mathrm{Ca} * \mathrm{P}(\mathrm{mg} 2 / \mathrm{dl} 2)$ & $49.02 \pm 10.48$ & $48.56 \pm 11.68$ & $56.97 \pm 13.50 * \#$ \\
\hline iPTH(pg/ml) & $567.72 \pm 612.72$ & $357.37 \pm 299.47$ & 838.18 $\pm 701.32 \#$ \\
\hline SpKt/V & $1.52 \pm 0.18$ & $1.51 \pm 0.73$ & $1.59 \pm 0.42$ \\
\hline Klotho protein(ng/L) & $423.93 \pm 63.15$ & $410.23 \pm 55.65$ & $387.21 \pm 37.79^{*}$ \\
\hline
\end{tabular}

Note: BMI, body mass index; Fer, ferritin. Compared with the non-calcification group, ${ }^{*} \mathrm{P}<0.05, \quad{ }^{* *} \mathrm{P}<0.01$; Compared to mild to moderate calcification group, \# $\mathrm{P}<0.05$.

Table 2: Comparison of cardiac structure and function in three groups of patients.

\begin{tabular}{|c|c|c|c|c|}
\hline Indicators & Non-Calcification Group & Mild to Moderate Calcification Group & Severe Calcification Group & P Value \\
\hline LVDd(mm) & $47.30 \pm 4.24$ & $48.81 \pm 6.02$ & $47.06 \pm 4.40$ & 0.515 \\
\hline LVDs(mm) & $29.674 \pm .03$ & $31.13 \pm 4.67$ & $29 \pm 2.70$ & 0.303 \\
\hline IVST (mm) & $11.48 \pm 1.35$ & $13.25 \pm 1.34^{* *}$ & $13.33 \pm 1.50^{* *}$ & 0 \\
\hline LVPWT (mm) & $10.30 \pm 1.18$ & $11.38 \pm 1.26^{* *}$ & $11.07 \pm 1.03^{*}$ & 0.008 \\
\hline EF (\%) & $66.64 \pm 5.68$ & $65.69 \pm 3.55$ & $68.07 \pm 2.84$ & 0.368 \\
\hline E/A>1[n (\%)] & $4(12.12 \%)$ & $1(6.25 \%)$ & $2(13.33 \%)$ & 0.76 \\
\hline LVMI(g/m2) & $151.38 \pm 33.65$ & $176.88 \pm 43.38^{*}$ & $173.61 \pm 29.06^{*}$ & 0.031 \\
\hline
\end{tabular}

Note: compared with the non-calcification group, ${ }^{*} \mathrm{P}<0.05, \mathrm{P}<0.01$.

\section{Relationship Between Coronary Artery Calcification Score and Cardiac Structure and Function}

64 patients were divided into non-calcification group, mild to moderate calcification group and severe calcification group according to CACs. The results showed that there were differences in IVST, LVPWT and LVMI among those groups. The levels of IVST, LVPWT and LVMI in mild to moderate calcification group and severe calcification group were significantly higher than those in non-calcification group. Correlation analysis showed that CACs was positively correlated with LVPWT and LVMI ( $r=0.307,0.348,0.014$, 0.005). Multiple stepwise regression analysis showed that CACs was the influencing factor of LVMI $(\beta=3.836)$ (Table 2$)$.

\section{Influencing Factors of Coronary Artery Calcification}

Compared with the non-calcification group, the average age of patients in the calcification group was older, the prevalence rate of diabetes was higher, the levels of BMI and blood CRP were higher, and there was statistical difference. The levels of serum albumin and Klotho protein in high calcification group were lower than those in non-calcification group and mild to moderate calcification group, while the levels of triglyceride, serum phosphorus and calcium-phosphorus product in high calcification group were significantly higher than those in the other two groups. The level of iPTH in severe calcification group was higher than that in mild and moderate calcification group, but there was no significant 
difference between severe calcification group and non-calcification group (Table 1).

The results of correlation analysis showed that the level of CACs was positively correlated with age, BMI, serum phosphorus, calcium-phosphorus product, CRP and triglyceride $(=0.383$, $0.319,0.281,0.278,0.299,0.286, \mathrm{P}=0.002,0.01,0.024,0.026$, $0.016,0.022)$, and negatively correlated with albumin and Klotho protein( $\mathrm{r}=-0.334,-0.299, \quad \mathrm{P}=0.007, \quad 0.016)$.Multiple stepwise regression analysis was used, with logarithmic transformation of CACs, discriminant criteria as $\mathrm{P}<0.05$, and excluded criteria as $\mathrm{P}>0.1$. The results showed that age, albumin, triglyceride and Klotho protein levels were the main factors affecting coronary artery calcification in young and middle-aged patients. Age and triglyceride were risk factors, while albumin and Klotho protein were protective factors (Table $1 \& 3$ ).

Table 3: Multiple linear regression analysis of CACs and related factors.

\begin{tabular}{|c|c|c|c|c|c|}
\hline Variable & B & Standard Error & Standardize B & T Value & P Value \\
\hline Age & 0.112 & 0.033 & 0.35 & -416 & 0.001 \\
\hline Albumin & -0.383 & 0.121 & -0.325 & -3.15 & 0.001 \\
\hline Triglyceride & 0.531 & 0.15 & 0.358 & 3.527 & 0.003 \\
\hline Klotho protein & -0.014 & 0.005 & -0.273 & -2.736 & 0.008 \\
\hline
\end{tabular}

\section{Discussion}

\section{Prevalence Rate of Coronary Artery Calcification in Young and Middle-Aged Hemodialysis Patients}

Cardiovascular disease is the most important and serious complication in patients with chronic kidney disease. More than $50 \%$ of maintenance hemodialysis patients die of cardiovascular disease. Coronary artery calcification can be present at any stage of the development of atherosclerotic plaques and is closely related to age. The sensitivity of coronary artery calcification for coronary heart disease in young and middle-aged people is $50 \%$, and the specificity is as high as $100 \%$, indicating that once the coronary artery is calcified, there may be coronary artery stenosis, increasing the occurrence of cardiovascular events. Goodman et al reported that $87.5 \%$ of the young dialysis patients (20-30 years old) had coronary artery calcification (14/16), while only $5 \%$ of the normal population had coronary artery calcification (3/60) [5].

In this study, it was found that the incidence of coronary artery calcification in young and middle-aged hemodialysis patients (average age of 48.63 years) was $48.44 \%$ (31/64), which was basically consistent with previous report [6]. It has been reported that the degree of left ventricular hypertrophy in patients with coronary artery calcification is higher than that in patients without coronary artery calcification. Guerin [7] found that EF and E/A decreased significantly in patients with CAC. This study also showed that IVST, LVPWT and LVMI were significantly increased in CAC group, and CACs was the influencing factor of LVMI. The underlying mechanism could be that arterial calcification leads to the increase of vascular resistance, which affects ventricular stroke volume and finally leads to changes in cardiac function and structure. However, there was no difference between EF and E/A groups, which may be explained by their compensatory cardiac function at young age.
It is still controversial regarding of related factors of coronary artery calcification in maintenance dialysis patients. Our study showed that the prevalence of diabetes was significantly higher in patients with coronary artery calcification, but there was no difference in the prevalence of hypertension, systolic blood pressure and diastolic blood pressure. This discrepancy could be explained by the use of antihypertensive drugs and good self-management in young patients considering that patient compliance was better in young patients. It was reported that coronary vascular calcification could be irrelevant to intradialytic blood pressure abnormalities in patients with end-stage renal disease on dialysis [8]. Some other studies found a relationship between vascular calcification and intradialytic hypotension $[9,10]$. Although the conflicting results are noteworthy, it may be the result of differences in the arteries used.

\section{The Risks of Coronary Artery Calcification}

Mcullough PA [11] systematically reviewed the results of 30 studies in the past 20 years and concluded that age was one of the most hazardous factors affecting coronary artery calcification. Our study also came to the same conclusion. Malnutrition-inflammationatherosclerosis syndrome ((MIA)) promotes atherosclerosis and coronary calcification in patients with CKD. Our study showed that coronary artery calcification was associated with CRP and Albumin. Regression analysis showed that albumin is one of the risk factors of coronary artery calcification. At the same time, we found that the BMI level in the calcification group was higher than that in the non-calcification group, which was contradictory to the mentioned theory, which could be explained by higher proportion of males in the calcification group. We also found that the level of triglyceride in severe calcification group was significantly higher than that in non-calcification group and mild to moderate calcification group, which was basically consistent with the study by Tamashiro [12]. 
The role of Klotho in artery calcification in young and middleaged hemodialysis patients and its potential use

Klotho gene is a newly discovered anti-aging gene. It was discovered by Kuro-o in 1997 and showed a downward trend with the increase of age. Klotho gene is mainly expressed in kidney and brain, including membrane-bound protein and secretory protein. Among them, the secretory Klotho protein is the dominant. We measured the secretory klotho protein, and found that the serum Klotho protein in the high calcification group was lower than that in the other two groups. Regression analysis showed that it was a protective factor for coronary artery calcification, which was basically consistent with the study by Semba [13].

The decrease in levels of Klotho may also indicate heart hypertrophy in hemodialysis patients [14]. Low levels of Klotho in patients with chronic kidney disease can also promote vessel calcification and salt-dependent hypertension [15,16]. Animal studies indicate a nephroprotective role of Klotho probably by influence on the activity of renin-angiotensin-aldosterone system [17]. This may provide a new target for the treatment of calcium and phosphorus metabolism. In short, the incidence of coronary artery calcification in young and middle-aged dialysis patients is high. Early screening and early detection, early intervention of calcium and phosphorus metabolism disorders, lipid metabolism disorders, malnutrition and other risk factors could be a focus of clinicians to improve the long-term prognosis of hemodialysis patients.

\section{Limitations}

There are many shortcomings of our study: no healthy control group, some patients took calcium and phosphorus drugs, which may result in a certain degree of bias. We did not follow-up those patients, nor probe the underlying mechanisms. Further study is needed in future to analysis the influencing factors of coronary artery calcification in young and middle-aged maintenance hemodialysis patients.

\section{Conclusion}

The prevalence of coronary artery calcification is high in young and middle-aged patients of maintenance hemodialysis. Klotho may be a new treatment target for the coronary artery calcification.

\section{Availability of Data and Materials}

The datasets used and/or analysed during the current study are available from the corresponding author on reasonable request.

\section{Acknowledgments}

We thank A-cha for date collecting, Ye bairu for date collecting. We would like to thank study participants for their time and contributions to the study.

\section{Funding Sources}

Supported by foundation of Zhejiang educational committee, No Y202044370

\section{Ethics Declarations}

Ethics approval and consent to participate.

Ethical issues (Including plagiarism, informed consent, misconduct, data fabrication and/or falsification, double publication and/or submission, redundancy, etc.) have been completely observed by the authors. Written informed consent was obtained from all patients in study. The study was approved by the Ethics Committee of the Second affiliated hospital of Wenzhou Medical University, February 2015.

\section{Contributions}

Shi zhen and Zheng shu-bei contributed writing and revisions to the manuscript and led data collection respectively. Jin Lingwei conducted analysis, wrote the first draft of the paper, and contributed revisions to the manuscript. Zhou Zhi-hong and Huang Wen co-led data collection activities respectively, conceived of the study, wrote the study proposal and contributed revisions to the manuscript. All authors read and approved the final manuscript.

\section{Conflict of Interest Statement}

We declare that we have no conflicts of interest. All authors made a substantial contribution to the information or material submitted for publication. All read and approved the final manuscript.

\section{References}

1. Levin A (2003) Clinical epidemlology of cardiovascular disease in chronic kidney disease prior to dialysis. Semin Dial 16(1): 101-105.

2. Gue rin AP, London GM, Marchais S, F Metivier (2000) Arterial stiffening and vascular calcification in endstage renal disease. Nephrol Dial Transplant 15(7): 1014-1021.

3. Hu MC, Shi M, Zhang J, Henry Quiñones, Makoto Kuro-o, et al. (2010) Klotho deficiency is an early biomarker of renal ischemia reperfusion injury and its replacement is protective. Kidney Int 78(12): 1240-1251.

4. Zheng Shubei, Zheng Yu, Jin Lingwei, Zhihong Zhou, Zhanyuan Li, et al. (2018) Relationship between Serum Soluble Klotho Protein and Coronary Artery Calcification and Prognosis in Patients on Maintenance Hemodialysis. Iran J Public Health 47: 510-518.

5. Goodman William G, Jonahtan Coldin, B D Kuizon, C Yoon, B Gales, et al. (2000) Coronary artery calcification in young adults with endstage renal disease who are undergoing dialysis. N Engl J Med 342: 1472-1483.

6. Barreto DV, Barreto FC, Carvalho AB, Lilian Cuppari, Miguel Cendoroglo, et al. (2005) Coronary calcification in hemodialysis patients: the contribution of traditional and uremia-related risk factors. Kidney Int 67(4): 1576-1582.

7. Guerin AP, London GM, Marchais SJ (2000) Arterial stiffening and vascular calcifications in end-stage renal disease. Nephrol Dial Tran splant 15: 1014-1021.

8. Yeo Seongyup, Moon Ji In, Shin Jungho, Jin Ho Hwang, Iksung Cho, et al. (2020) Impacts of Coronary Artery Calcification on Intradialytic Blood Pressure Patterns in Patients Receiving Maintenance Hemodialysis. Chonnam Med J 56(1): 27-35.

9. Cho A, Lee YK, Oh J, Yoon JW, Shin DH, et al. (2017) The relationship between intradialytic hypotension and vascular calcification in hemodialysis patients. PLOS One 12(10): e0185846.

10. Kim SY, Hong YA, Yoon HE, Chang YK, Yang CW, et al. (2016) Vascular calcification and intradialytic hypotension in hemodialysis patients: 
clinical relevance and impact on morbidity and mortality. Int J Cardiol. 217: 156-160.

11. Mcullough PA, Sandberg KR, Dumler F, Jose E Yanez (2004) Determinants of coronary vascular-calcification in patients with chronic kidney disease and end stage renal disease asystematic review. J Nephrol 17(2): 205-215.

12. Tamashiro M, Lseki K, Sunaqawa O, T Inoue, S Higa, et al. (2001) Significant association between the progression of coronary artery calcification and dyslipidemia in patients on chronic hemodialysis. Am J Kidney Dis 38(1): 64-69.

13. Semba RD, Cappola AR, Sun K, Stefania Bandinelli, Mansi Dalal, et al (2011) Plasma Klotho and cardiovascular disease in aldult. J Am Geriatr Soc 59(9): 1596-1601.

14. Zbroch Edyta, Bazyluk Angelika, Malyszko Jolanta (2020) The Serum Concentration of Anti-Aging Proteins, Sirtuin1 and $\alpha$ Klotho in Patients with End-Stage Kidney Disease on Maintenance Hemodialysis. Clin Interv Aging 15: 387-393.

15. Kalaitzidis RG, Duni A, Siamopoulos KC (2016) Klotho, the Holy Grail of the kidney: from salt sensitivity to chronic kidney disease. Int Urol Nephrol 48(10): 1657-1666.

16. Zhou L, Mo H, Miao J, Dong Zhou, Roderick J Tan, et al. (2015) Klotho ameliorates kidney injury and fibrosis and normalizes blood pressure by targeting the renin-angiotensin system. Am J Pathol 185(12): 32113223.

17. Gao D, Zuo Z, Tian J, Quaisar Ali, Yi Lin, et al. (2016) Activation of SIRT1 attenuates klotho deficiency-induced arterial stiffness and hypertension by enhancing AMP-activated protein kinase activity. Hypertension 68(5): 1191-1199. 